\title{
Performance Analysis of User Pairing Algorithm in Full-Duplex Cellular Networks
}

\author{
Wonjong Noh, ${ }^{1}$ Wonjae Shin, ${ }^{2}$ and Hyun-Ho Choi $^{3}$ \\ ${ }^{1}$ Samsung Electronics Co., Ltd., Suwon 16677, Republic of Korea \\ ${ }^{2}$ Department of Electrical and Computer Engineering, Seoul National University, Seoul 08826, Republic of Korea \\ ${ }^{3}$ Department of Electrical, Electronic and Control Engineering, Hankyong National University, Anseong 17579, Republic of Korea
}

Correspondence should be addressed to Hyun-Ho Choi; hhchoi@hknu.ac.kr

Received 9 December 2016; Accepted 5 March 2017; Published 23 March 2017

Academic Editor: Hideyuki Takahashi

Copyright (C) 2017 Wonjong Noh et al. This is an open access article distributed under the Creative Commons Attribution License, which permits unrestricted use, distribution, and reproduction in any medium, provided the original work is properly cited.

\begin{abstract}
In a full duplexing (FD) wireless cellular network, a base station operates in FD mode, while the downlink (DL) and uplink (UL) users operate in half duplexing (HD) mode. Thus, the downlink and uplink transmissions occur simultaneously so that interuser interference from a UL to a DL user occurs. In an FD network, the main challenge to minimize the interuser interference is $u s e r$ pairing, which determines a pair of DL and UL users who use the same radio resource simultaneously. We formulate a nonconvex optimization problem for user pairing to maximize the cell throughput. Then, we propose a heuristic user pairing algorithm with low complexity. This algorithm is designed such that the DL user having a better signal quality has higher priority to choose its paired UL user for throughput maximization. Thereafter, we conduct theoretical performance analysis of the FD cellular system based on stochastic geometry and analyze the impact of the user paring algorithm on the performance of the FD cellular system. Results show that the FD system that uses the proposed user pairing algorithm effectively reduces the interuser interference and approaches optimal performance. It also considerably outperforms the FD system using a random user pairing and almost doubles the conventional HD system in terms of cell throughput.
\end{abstract}

\section{Introduction}

In traditional wireless communication, the duplexing mode for transmitting and receiving signals at a node commonly is half duplex (HD), such as frequency division duplexing (FDD) and time division duplexing (TDD). As another duplexing mode, full duplexing (FD) has recently received considerable attention because FD enables a node to transmit and receive signals simultaneously on the same frequency so that spectral efficiency can be improved twice in theory. However, the main challenge in applying the FD to wireless networks is the manner in which one can handle cochannel interference not only between the transmit (Tx) and receive $(\mathrm{Rx})$ antennas at the base station (BS), but also between the uplink (UL) and downlink (DL) communication users. The cochannel interference between the Tx and $\mathrm{Rx}$ at the same station is called self-interference, whereas that between the different stations is called interuser interference.
To address self-interference, a smart code that divides the transmitting and receiving signals has been invented and a code division duplexing (CDD) system using this coding technique has been studied $[1,2]$. Then, the CDD system has been shown to achieve twice the spectral efficiency of the HD system [3]. In addition, advanced antenna cancellation techniques that cancel the self-interference between $\mathrm{Tx}$ and $\mathrm{Rx}$ on the radio frequency $(\mathrm{RF})$ band have been proposed and real performances by implementation have been demonstrated [4-6]. Unlike these physical layer solutions, scheduling on the medium access control (MAC) layer has been another research issue studied in order to tackle the self-interference problem in an FD system. The self-interference problems at the wireless relay node with the FD have been resolved by dynamic resource allocation schemes [7-9]. In a practical FD cellular system in which multiple Rxs of the BS are distributed at a distance with the Tx of BS, scheduling algorithms that determine both subcarrier allocation and the communication 
direction between UL and DL users have been designed to maximize cell throughput [10].

To address interuser interference in FD systems, various algorithms including resource allocation, scheduling, user pairing, and their optimization have been studied to improve system performance with respect to cell throughput, cell coverage, and outage probability. Some initial studies were conducted to examine various resource allocation-related problems in FD cellular networks [11-13]. Resource block (RB) allocation was considered in [11] for an FD cellular network, and interference between users was reduced as a result. In [12], a two-user FD cellular network was considered and a noncooperative game was proposed for radio resource allocation in such networks. In [13], a power efficient resource allocation algorithm was designed to achieve total transmit power minimization in an FD cellular network.

In addition, various scheduling algorithms for an FD system that consider nonconvex and combinatorial optimization problems were studied in [14]. The Janus protocol in [15] also considers a scheduling algorithm for scheduling a transmission mode (HD or FD mode) to maximize in-band FD transmission opportunity, while maintaining fair channel access for all nodes. Recently, a MAC scheduling algorithm that employs cooperative FD relays (FDRs) was proposed in [16]. This study revealed that additional throughput can be achieved by using cooperative FDRs. In [17], joint user scheduling and channel allocation for cellular networks was considered and a suboptimal heuristic algorithm with low computational complexity was proposed. Furthermore, two heuristic user pairing algorithms were designed for throughput maximization and outage minimization, respectively [18]. Simulation results showed that these two algorithms approach optimal performance under the assumption of no residual self-interference at the FD BS. However, up-to-date studies and theoretical performance analysis on user pairing algorithms in FD cellular network environments have not been thoroughly conducted. Compared with [18], our study newly conducts theoretical analysis of the FD cellular system based on stochastic geometry after adopting the user pairing algorithm previously proposed and then investigates the theoretical performance of FD cellular networks according to various system parameters, which have not been considered in [18].

In this study, we consider FD wireless cellular networks in which the BS operates in FD mode while DL and UL users operate in HD mode. Because this FD system induces severe interuser interference from UL to DL users, the main concern in maximizing system performance is user pairing, which determines a pair of DL and UL users that use the same radio resource simultaneously. We formulate the optimization problems for user pairing to maximize cell throughput. Solving these optimization problems by means of exhaustive search is highly complex; thus we propose a suboptimal user pairing algorithm with low computation complexity for practical use. In a heuristic manner, the proposed user pairing algorithm considers the signal quality of users and the direct channel between users. Thereafter, we perform theoretical analysis of the FD cellular system based on stochastic geometry and investigate the effects of adopting the user pairing algorithm. Analysis and simulation show that the FD system in conjunction with the proposed user pairing algorithm approaches optimal performance and greatly improves cell throughput compared to the conventional HD system.

The rest of this paper is organized as follows. Section 2 describes the system model of the considered FD wireless cellular network. In Section 3, user pairing algorithm is proposed for throughput maximization. Section 4 provides stochastic geometry-based analysis of the FD cellular network. In Section 5, analysis and simulation results are given and the performance gain of the proposed pairing algorithm is verified. Conclusion remarks are provided in Section 6.

\section{System Description}

We consider a cellular network composed of multiple cells in which each cell is surrounded by $N$ neighbor cells, as shown in Figure 1. The BS works in FD mode while DL and UL users work in HD mode due to the limited processing ability of user terminals. We assume that a total of $2 M$ users are present in a cell and that both the number of UL users and the number of DL users are $M$ equally. Moreover, one transmission frame is divided into $M$ time slots and each user is allowed to use only one time slot within a frame to transmit or receive data. This time slot allocation corresponds to the manner of roundrobin scheduling of radio resources and thus provides some degree of fairness for all users [19].

Because the DL and UL users transmit signals on the same resource simultaneously, the DL user experiences three different types of interferences from (i) the UL user scheduled in the same slot in the serving cell, (ii) the neighboring BSs, and (iii) the UL users using the same slot in neighboring cells. Therefore, the received signal at DL user $i$ is expressed as

$$
\begin{aligned}
y_{i}= & \sqrt{P_{0}} h_{d, i} x_{i}+\underbrace{\sqrt{P_{j}} \varphi_{i j} x_{j}}_{\text {interuser intf. }}+\underbrace{\sum_{n=1}^{N} \sqrt{P_{0}} g_{i n} \widehat{x}_{n}}_{\text {intf. from neighbor BSs }} \\
& +\underbrace{\sum_{n=1}^{N} \sqrt{P_{n}} \varphi_{i n} \check{x}_{n}}_{\text {intf. from neighbor UL users }}+n_{i},
\end{aligned}
$$

where $P_{0}$ and $P_{j}$ are the transmission power of the BS and UL user $j$, respectively. The variables $x_{i}, x_{j}$, and $x_{n}$ denote the signals sent from the serving $\mathrm{BS}_{0}$, the UL user $j$ using the same time slot, and the $n$th neighboring cell, respectively. As illustrated in Figure 1, the channel coefficients $h_{d, i}$ and $\varphi_{i j}$ correspond to the links to DL user $i$ from its serving $\mathrm{BS}$ and the UL user $j$, respectively. In addition, the channel coefficients $g_{\text {in }}$ and $\varphi_{\text {in }}$ correspond to the links to DL user $i$ from the BS and UL user in the $n$th neighboring cell, respectively. Here, all the channel coefficients are assumed to be $\mathscr{C} \mathscr{N}\left(0, \sigma^{2} / d_{i j}^{\alpha}\right)$, where $d_{i j}$ is the transmission distance of the corresponding ij link and $\alpha$ denotes the path loss exponent. Finally, $n_{i}$ is the additive white Gaussian noise with zero mean and $N_{0}$ variance. 


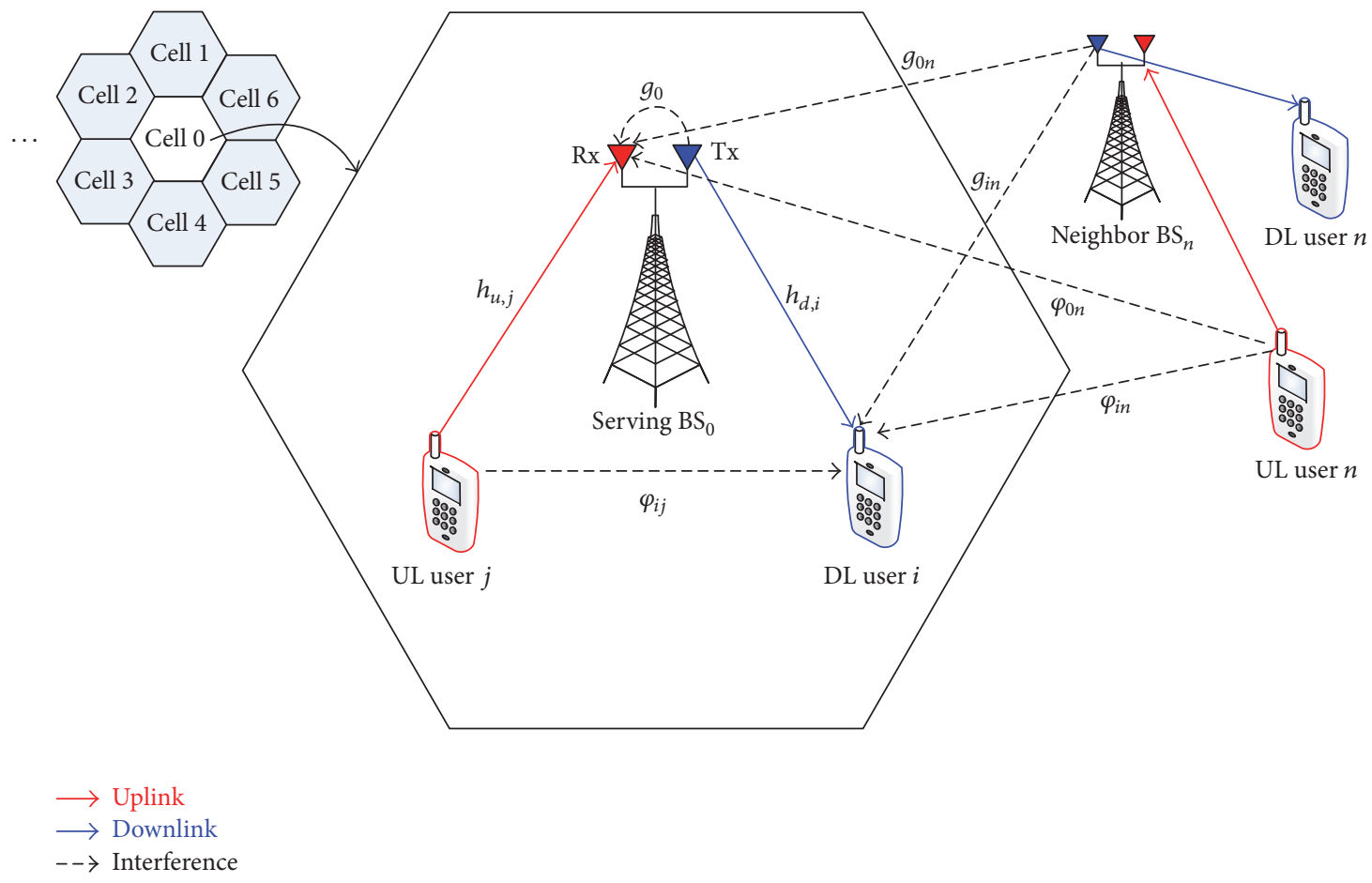

FIGURE 1: Considered full-duplex cellular networks.

In addition, the $\mathrm{Rx}$ of the $\mathrm{BS}$ experiences three kinds of interferences from (i) the Tx of the serving BS, (ii) the neighboring BSs, and (iii) the UL users using the same slot in neighboring cells. Therefore, the signal received from the $\mathrm{UL}$ user $j$ at the $\mathrm{Rx}$ of the serving $\mathrm{BS}_{0}$ is expressed as

$$
\begin{aligned}
y_{j}= & \sqrt{P_{j}} h_{u, j} x_{j}+\underbrace{\sqrt{P_{0}} g_{0} x_{i}}_{\text {self-interference }}+\underbrace{\sum_{n=1}^{N} \sqrt{P_{0}} g_{0 n} \widehat{x}_{n}}_{\text {intf. from neighbor BSs }} \\
& +\underbrace{\sum_{n=1}^{N} \sqrt{P_{n}} \varphi_{0 n} \check{x}_{n}}_{\text {intf. from neighbor UL users }}+n_{0},
\end{aligned}
$$

where $h_{u, j}, g_{0}, g_{0 n}$, and $\varphi_{0 n}$ represent the channel coefficients corresponding to the links from the UL user $j$, the Tx of the serving $\mathrm{BS}_{0}$, the $n$th neighboring $\mathrm{BS}$, and the $n$th neighboring UL user, respectively.

In the FD system, the $\mathrm{Rx}$ of the $\mathrm{BS}$ experiences strong interferences from the Txs of both the serving and neighboring BSs (relatively, the interferences from the Txs of UL users in the neighboring cells are weak because of their small transmission power and the long distance to the neighboring BS [10]). However, the BS with FD function can eliminate these kinds of interferences by using the antenna's cancellation techniques and channel estimation techniques $[5,10]$. For the purpose of channel estimation, we assume that the $\mathrm{Rx}$ at the BS employs pilot symbols, which are transmitted from the Txs of both the serving $\mathrm{BS}_{0}$ and neighboring BSs. Although the received interference power is very strong compared to the receiving signal strength at the $\mathrm{BS}$, it can be reduced to the same level as the receiver noise floor by applying analog and digital cancellation techniques sequentially [4-6]. Moreover, by adopting a proper channel estimation technique, such as least-square and minimum mean square error, we can estimate the channel coefficients $g_{0}$ and $g_{0 n}$ [20] (details about channel estimation techniques are out of the scope of this study; the channel estimation at the BS is assumed to be perfect for the practicality of the FD). Based on this interference channel estimation and the knowledge of the signals sent from the Txs of the serving $\mathrm{BS}_{0}$ and neighboring BSs through the wired backhaul, the Rx of the serving $\mathrm{BS}_{0}$ can eliminate the corresponding interference parts from the received signal. After interference cancellation, the postprocessed signal of UL user $j$ is expressed as

$$
\begin{aligned}
\widehat{y}_{j}= & \sqrt{P_{j}} h_{u, j} x_{j}+\sqrt{P_{0}}\left(g_{0}-\widehat{g}_{0}\right) x_{i} \\
& +\sum_{n=1}^{N} \sqrt{P_{0}}\left(g_{0 n}-\widehat{g}_{0 n}\right) \widehat{x}_{n}+\sum_{n=1}^{N} \sqrt{P_{n}} \varphi_{0 n} \check{x}_{n}+n_{0},
\end{aligned}
$$

where $\widehat{g}_{0}$ and $\widehat{g}_{0 n}$ denote the estimated channel coefficients.

From (1) and (3), the signal-to-interference-plus-noise ratios (SINRs) of the received signals $y_{i}$ and $\hat{y}_{j}$ are, respectively, given by

$$
\gamma_{i}=\frac{P_{0}\left\|h_{d, i}\right\|^{2}}{P_{j}\left\|\varphi_{i j}\right\|^{2}+\sum_{n=1}^{N} P_{0}\left\|g_{i n}\right\|^{2}+\sum_{n=1}^{N} P_{n}\left\|\varphi_{i n}\right\|^{2}+N_{0}},
$$




$$
\begin{aligned}
& \gamma_{j} \\
& =\frac{P_{j}\left\|h_{u, j}\right\|^{2}}{P_{0}\left\|g_{0}-\widehat{g}_{0}\right\|^{2}+\sum_{n=1}^{N} P_{0}\left\|g_{0 n}-\widehat{g}_{0 n}\right\|^{2}+\sum_{n=1}^{N} P_{n}\left\|\varphi_{0 n}\right\|^{2}+N_{0}} .
\end{aligned}
$$

Consequently, the rates (i.e., spectral efficiencies) of DL user $i$ and UL user $j$ are given by

$$
\begin{aligned}
& R_{i}=\log _{2}\left(1+\gamma_{i}\right), \\
& R_{j}=\log _{2}\left(1+\gamma_{j}\right) .
\end{aligned}
$$

Finally, the cell throughput of FD system is expressed as

$$
S_{\mathrm{FD}}=\sum_{i=1}^{M} R_{i}+\sum_{j=1}^{M} R_{j}
$$

In the conventional HD system (e.g., TDD system) in which neither self-interference nor interuser interference exists, the SINRs of DL user $i$ and UL user $j$ are, respectively, expressed as

$$
\begin{aligned}
& \gamma_{i}^{\mathrm{HD}}=\frac{P_{0}\left\|h_{d, i}\right\|^{2}}{\sum_{n=1}^{N} P_{0}\left\|g_{i n}\right\|^{2}+N_{0}}, \\
& \gamma_{j}^{\mathrm{HD}}=\frac{P_{j}\left\|h_{u, j}\right\|^{2}}{\sum_{n=1}^{N} P_{n}\left\|\varphi_{0 n}\right\|^{2}+N_{0}} .
\end{aligned}
$$

Therefore, the cell throughput of HD system is expressed as

$$
S_{\mathrm{HD}}=\frac{1}{2}\left\{\sum_{i=1}^{M} \log _{2}\left(1+\gamma_{i}^{\mathrm{HD}}\right)+\sum_{j=1}^{M} \log _{2}\left(1+\gamma_{j}^{\mathrm{HD}}\right)\right\} \text {. }
$$

\section{Proposed User Pairing Algorithm}

Based on (4) and (5), the parameters that we can control in the FD system are $P_{j}$ and $\varphi_{i j}$. The other parameters are given based on the node position or are generally fixed. Here, $\varphi_{i j}$ is controlled by the user pairing. Thus, the user pairing influences the DL SINR and cell throughput. Our objective is to design a novel user pairing algorithm to optimize cell throughput.

We denote by $(i, j)$ a user pair in which DL user $i$ and UL user $j$ are allocated to the same time slot. Possible user pairs are derived by choosing a number $i$ in $\{1,2, \ldots, M\}$ and a number $j$ in $\{1,2, \ldots, M\}$ under the constraint that the chosen number cannot be selected again. Let $\Pi$ denote the set of all possible user pairs. For example, for $M=3$, $\Pi$ becomes

$$
\begin{gathered}
\Pi=\{\{(1,1),(2,2),(3,3)\},\{(1,1),(2,3),(3,2)\}, \\
\quad\{(1,2),(2,1),(3,3)\},\{(1,2),(2,3),(3,1)\}, \\
\\
\{(1,3),(2,1),(3,2)\},\{(1,3),(2,2),(3,1)\}\},
\end{gathered}
$$

where six configurations exist. For a general $M$, the number of possible configurations for user pairing is given by $M$ !. We denote by $\pi_{k}$ the $k$ th element of $\Pi$, where $k=1,2, \ldots, M$ !.
For the purpose of maximizing cell throughput, the optimization problem is formulated as

$$
\begin{aligned}
\pi_{k}=\arg \max & \sum_{(i, j) \in \pi_{k}} R_{i}+R_{j} \\
\text { s. t. } & P_{j}=P_{\max } \\
& \\
&
\end{aligned}
$$

where $P_{\max }$ is the maximum transmission power of the UL user. In this problem, every UL user should set its transmission power $P_{j}$ to $P_{\max }$ to maximize its UL throughput. To solve this nonconvex and combinatorial optimization problem, we must search the total $M$ ! configurations for the user pairs. This computation complexity is $O(M !)$, which is surprisingly high with a large $M$ (i.e., a large number of users). Therefore, we propose a suboptimal pairing algorithm with low complexity for practical use.

To design a user pairing algorithm, we can obtain insight from (4) and (5). As shown in (5), the SINR of UL user $\gamma_{j}$ is not affected by DL users. If we assume that the channel estimation and interference cancellation are perfect and the interference from neighbor UL users is negligible, $\gamma_{j}$ depends on mainly UL channel $h_{u, j}$. By contrast, the SINR of DL user $\gamma_{i}$ depends not only on the DL channel, $h_{d, i}$, but also on the direct channel from UL user $j$ to DL user $i, \varphi_{i j}$. Therefore, the user pairing influences only the SINR of DL user $\gamma_{i}$. To maximize the sum rate of all users, having the user with a better signal quality receive less interference is reasonable [21]. Therefore, we have the DL user with a better signal quality (i.e., a higher value of $\gamma_{i}^{\mathrm{HD}}$ ) choose first the UL user with a smaller $\varphi_{i j}$ as its partner. This approach improves the SINR of the DL user with a better signal quality more by reducing more interference from the UL user, and it eventually increases the sum rate of all users.

The proposed user pairing algorithm is given as Algorithm 1. First, the transmit power of all UL users is initialized to their available maximum power, $P_{\max }$, in order to maximize the UL SINR at the BS. Moreover, the flag indicates whether the UL user is selected for user pairing and is initialized to 0 for all $j$. Thereafter, the algorithm sorts the DL user in order from the highest to lowest DL SINR value and then runs based on these sorted DL users. For DL user $i$, the pairing algorithm measures the direct channel $\varphi_{i j}$ from the unpaired UL user $j$ to the DL user $i$. Then, the UL user having the smallest $\varphi_{i j}$ value among the unpaired UL users is selected for pairing. Accordingly, the flag of this UL user $j$ is set to 1 ; thus, the DL user $i$ and selected UL user $j$ are paired with each other. This pairing operation is repeated until all sorted DL users are paired with the remaining UL users. Note that the proposed algorithm depends on only one iteration and one search within each iteration for $M$ DL/UL users so that its computation complexity becomes $O\left(M^{2}\right)$.

\section{Performance Analysis}

4.1. Assumptions and Notations. For the stochastic geometrybased analysis, we assume a homogeneous cellular network that consists of macro-cell BSs and users. The BSs are 
(1) Initialize:

$$
\begin{aligned}
& P_{j} \leftarrow P_{\max }, j=1,2, \ldots, M \\
& U_{j} \leftarrow 0, j=1,2, \ldots, M \quad I^{*} \text { flag for selected or not }{ }^{*} /
\end{aligned}
$$

(2) Sort the DL users in order of high $\gamma_{i}^{\mathrm{HD}}$ value

(3) for $i=$ [sorted DL users] do

(4) Unpaired UL users $\leftarrow$ find $\left(U_{j}=0\right)$

(5) Measure $\varphi_{i j}$ from unpaired UL user $j$ to DL user $i$

(6) Choose the UL user $j$ with the smallest $\varphi_{i j}$ among unpaired UL users

(7) $U_{j} \leftarrow 1$

(8) Complete the user pair $(i, j)$

(9) end for

Algorithm 1: Proposed user pairing algorithm.

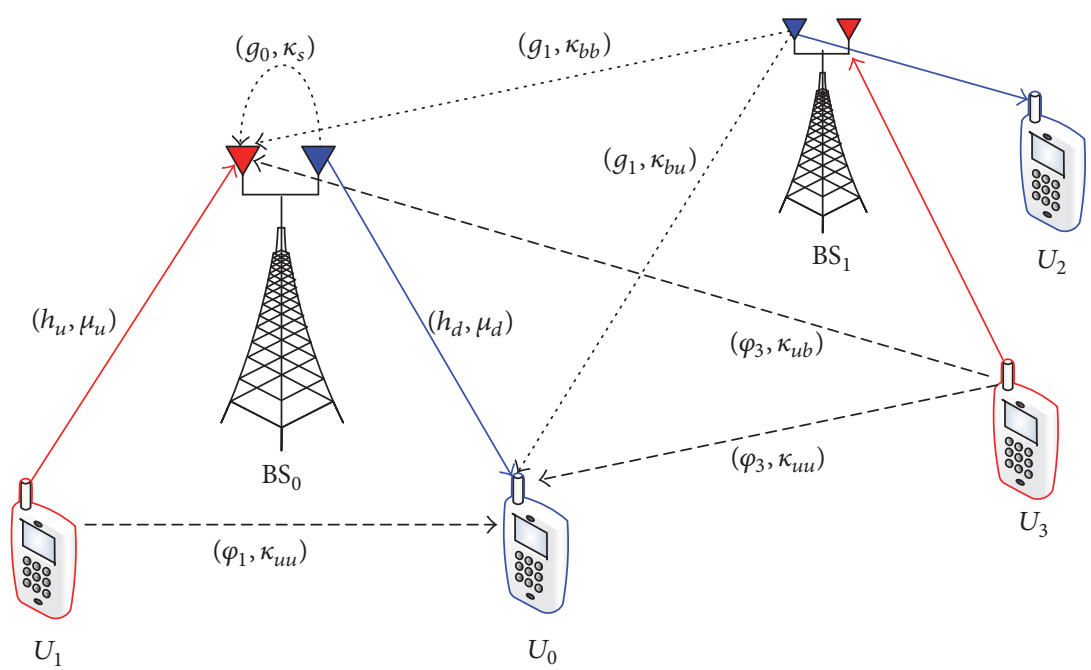

Figure 2: System model and notations.

arranged according to a homogeneous Poisson point process (PPP) $\Phi$ with intensity $\lambda$ in the Euclidean plane $[22,23]$. We also assume that DL and UL users are distributed according to an independent homogeneous PPP $\Omega$ with the same density $\lambda$ so that the FD BS schedules one UL user and one DL user concurrently during a given time/frequency resource unit $[23,24]$. Each user is connected to its closest BS.

Figure 2 shows the system model where a $\mathrm{BS}_{0}$ simultaneously serves its DL user $u_{0}$ and UL user $u_{1}$ through FD. While $u_{0}$ receives and $u_{1}$ transmits a signal from and to $\mathrm{BS}_{0}$ using $\mathrm{FD}, u_{0}$ receives interferences from $u_{1}, u_{3}$, and $\mathrm{BS}_{1}$, and $u_{1}$ receives interferences from $u_{3}, \mathrm{BS}_{0}$, and $\mathrm{BS}_{1}$. Normal line with $(h, \cdot)$ denotes the signal channel. Also, dotted line with $(g, \cdot)$ and dashed line with $(\varphi, \cdot)$ denote interference channels by the neighboring BSs and users, respectively. Here, we assume that a tagged BS and tagged user experience Rayleigh fading with unit mean and employ a constant transmit power of $1 / \mu$. In this case, the received power at a typical node at a distance $r$ from its BS is $h r^{-\alpha}$, where the random variable $h$ follows an exponential distribution with mean $1 / \mu$ (i.e., $h \sim \exp (\mu))$. It is denoted as $(h, \mu)$. Furthermore, all BSs and users experience a standard power loss propagation with path loss exponent $\alpha>2$ and additive and constant noise power of $\sigma^{2}$. All results are related to a single transmit antenna and single receive antenna, although future extension to multiple antennas is clearly desirable.

4.2. Downlink Rate in Full-Duplex. When a typical DL user, $u_{0}$, has a distance $r$ and fading strength $h_{d} \sim \exp \left(\mu_{d}\right)$ from its serving BS, the user $u_{0}$ receives a signal with a power of $h_{d} r^{-\alpha}$. However, the user $u_{0}$ experiences cumulative interferences $I_{b}$ and $I_{u}$ from neighbors, where $I_{b}$ is the sum of the received powers from all other BSs, each with distance $r_{i}$ and fading strength $g_{i} \sim \exp \left(\kappa_{b u}\right)$ to the user $u_{0}$ such that

$$
I_{b}=\sum_{i \in \Phi_{b}} g_{i} r_{i}^{-\alpha},
$$

where $\Phi_{b}$ is the set of all interfering BSs. In addition, $I_{u}$ is the sum of the received powers from all other UL users, each with distance $r_{i}$ and fading strength $\varphi_{i} \sim \exp \left(\kappa_{u u}\right)$ to the user $u_{0}$ such that

$$
I_{u}=\sum_{i \in \Omega_{u}} \varphi_{i} r_{i}^{-\alpha}
$$


where $\Omega_{u}$ is the set of all interfering users. The SINR of the typical DL user $u_{0}$ can then be expressed as

$$
\gamma_{\mathrm{DL}}=\frac{h_{d} r^{-\alpha}}{I_{b}+I_{u}+\sigma^{2}} .
$$

The average rate of the typical DL user $u_{0}$ is

$$
R_{\mathrm{DL}}(\lambda, \alpha)=\mathbf{E}_{I_{b}, I_{u}, r}\left[\ln \left(1+\gamma_{\mathrm{DL}}\left(I_{b}, I_{u}, r\right)\right)\right] .
$$

Because the probability that the closest BS has a distance $r$ is $e^{-\lambda \pi r^{2}} 2 \pi \lambda r$, (15) is calculated as

$$
\begin{aligned}
& R_{\mathrm{DL}}(\lambda, \alpha) \\
& =\int_{r>0} e^{-\pi \lambda r^{2}} \mathbf{E}_{I_{b}, I_{u}}\left[\ln \left(1+\frac{h_{d} r^{-\alpha}}{I_{b}+I_{u}+\sigma^{2}}\right) \mid r\right] \\
& \cdot 2 \pi \lambda r d r,
\end{aligned}
$$

where

$$
\begin{aligned}
\mathbf{E}_{I_{b}, I_{u}} & {\left[\ln \left(1+\frac{h_{d} r^{-\alpha}}{I_{b}+I_{u}+\sigma^{2}}\right) \mid r\right] } \\
= & \int_{t>0} \mathbf{P}_{I_{b}, I_{u}}\left[\ln \left(1+\frac{h_{d} r^{-\alpha}}{I_{b}+I_{u}+\sigma^{2}}\right)>t \mid r\right] d t \\
= & \int_{t>0} \mathbf{P}_{I_{b}, I_{u}}\left[h_{d}>r^{\alpha}\left(\sigma^{2}+I_{b}+I_{u}\right)\left(e^{t}-1\right) \mid r\right] d t \\
= & \int_{t>0} \mathbf{E}_{I_{b}, I_{u}}\left[\mathbf { P } \left[h_{d}\right.\right. \\
> & \left.\left.r^{\alpha}\left(\sigma^{2}+I_{b}+I_{u}\right)\left(e^{t}-1\right) \mid r, I_{b}, I_{u}\right]\right] d t \\
\mathbf{E}_{I_{b}, I_{u}} & {\left[\ln \left(1+\frac{h_{d} r^{-\alpha}}{I_{b}+I_{u}+\sigma^{2}}\right) \mid r\right] } \\
= & \int_{t>0} \mathbf{E}_{I_{b}, I_{u}}\left[e^{-\mu_{d} r^{\alpha}\left(\sigma^{2}+I_{b}+I_{u}\right)\left(e^{t}-1\right)}\right] d t \\
= & \int_{t>0} \mathbf{E}_{I_{b}, I_{u}}\left[e^{-\mu_{d} r^{\alpha} \sigma^{2}\left(e^{t}-1\right)} e^{-\mu r^{\alpha}\left(I_{b}+I_{u}\right)\left(e^{t}-1\right)}\right] d t \\
= & \int_{t>0} e^{-\mu_{d} r^{\alpha} \sigma^{2}\left(e^{t}-1\right)} \mathbf{E}_{I_{b}}\left[e^{-\mu_{d} r^{\alpha} I_{b}\left(e^{t}-1\right)}\right], \\
& \cdot \mathbf{E}_{I_{u}}\left[e^{-\mu_{d} r^{\alpha} I_{u}\left(e^{t}-1\right)}\right] d t \\
= & \int_{t>0} e^{-\mu_{d} r^{\alpha} \sigma^{2}\left(e^{t}-1\right)} \mathscr{L}_{I_{b}}\left(\mu_{d} r^{\alpha}\left(e^{t}-1\right)\right) \\
\cdot & \mathscr{L}_{I_{u}}\left(\mu_{d} r^{\alpha}\left(e^{t}-1\right)\right) d t .
\end{aligned}
$$$$
\text { since } h_{d} \sim \exp \left(\mu_{d}\right),
$$

In (17), the Laplace transforms of $I_{b}$ and $I_{u}$ are obtained as the following two lemmas, respectively.
Lemma 1. Laplace transform of $I_{b}$

$$
\begin{gathered}
\mathscr{L}_{I_{b}}\left(\mu_{d} r^{\alpha}\left(e^{t}-1\right)\right)=\exp \left(-\pi \lambda r^{2}\left(\beta_{d, b u}\left(e^{t}-1\right)\right)^{2 / \alpha}\right. \\
\left.\cdot \int_{\left(\beta_{d, b u}\left(e^{t}-1\right)\right)^{-2 / \alpha}}^{\infty} \frac{1}{1+x^{\alpha / 2}} d x\right) .
\end{gathered}
$$

Proof.

$$
\begin{aligned}
\mathscr{L}_{I_{b}}(s) & =\mathbf{E}_{I_{b}}\left[e^{-s I_{b}}\right] \\
& =\mathbf{E}_{\Phi}\left[\Pi_{i \in \Phi /\left\{b_{o}\right\}} \mathbf{E}_{g_{i}}\left[\exp \left(-s g_{i} R_{i}^{-\alpha}\right)\right]\right] \\
& =\mathbf{E}_{\Phi}\left[\Pi_{i \in \Phi /\left\{b_{o}\right\}} \frac{\kappa_{b u}}{\kappa_{b u}+s R_{i}^{-\alpha}}\right] \\
& =\exp \left(-\lambda \int_{\mathbb{R}^{2}}\left(1-\frac{\kappa_{b u}}{\kappa_{b u}+s x^{-\alpha}}\right) d x\right)
\end{aligned}
$$

where we employ 1-dimension integral by radius $v$,

$\mathscr{L}_{I_{b}}(s)=\exp \left(-2 \pi \lambda \int_{r}^{\infty}\left(1-\frac{\kappa_{b u}}{\kappa_{b u}+s v^{-\alpha}}\right) v d v\right)$.

Therefore,

$$
\begin{aligned}
& \mathscr{L}_{I_{b}}\left(\mu_{d} r^{\alpha}\left(e^{t}-1\right)\right) \\
& =\exp \left(-2 \pi \lambda \int_{r}^{\infty}\left(1-\frac{1}{1+\mu_{d} / \kappa_{b u}\left(e^{t}-1\right)(r / v)^{\alpha}}\right)\right. \\
& \cdot v d v) \quad \text { where we put } \frac{\mu_{d}}{\kappa_{b u}}=\beta_{d, b u} \\
& \mathscr{L}_{I_{b}}\left(\mu_{d} r^{\alpha}\left(e^{t}-1\right)\right) \\
& =\exp \left(-2 \pi \lambda \int_{r}^{\infty}\left(1-\frac{1}{1+\beta_{d, b u}\left(e^{t}-1\right)(r / v)^{\alpha}}\right) v d v\right) \\
& =\exp \left(-2 \pi \lambda \int_{r}^{\infty} \frac{1+\beta_{d, b u}\left(e^{t}-1\right)(r / v)^{\alpha}-1}{1+\beta_{d, b u}\left(e^{t}-1\right)(r / v)^{\alpha}} v d v\right) \\
& =\exp \left(-2 \pi \lambda \int_{r}^{\infty} \frac{\beta_{d, b u}\left(e^{t}-1\right)(r / v)^{\alpha}}{1+\beta_{d, b u}\left(e^{t}-1\right)(r / v)^{\alpha}} v d v\right) \\
& =\exp \left(-2 \pi \lambda \int_{r}^{\infty} \frac{\beta_{d, b u}\left(e^{t}-1\right)}{\beta_{d, b u}\left(e^{t}-1\right)+(v / r)^{\alpha}} v d v\right) \\
& \text { where we put } \frac{v^{2}}{r^{2}}=u,
\end{aligned}
$$$$
\mathscr{L}_{I_{b}}\left(\mu_{d} r^{\alpha}\left(e^{t}-1\right)\right)
$$

$$
=\exp \left(-\pi \lambda r^{2} \int_{1}^{\infty} \frac{\beta_{d, b u}\left(e^{t}-1\right)}{\beta_{d, b u}\left(e^{t}-1\right)+u^{\alpha / 2}} d u\right)
$$




$$
\begin{aligned}
& =\exp \left(-\pi \lambda r^{2} \int_{1}^{\infty} \frac{1}{1+\left(\left(\beta_{d, b u}\left(e^{t}-1\right)\right)^{-2 / \alpha} u\right)^{\alpha / 2}} d u\right) \\
& \text { where we put }\left(\beta_{d, b u}\left(e^{t}-1\right)\right)^{-2 / \alpha} u=x, \\
& \mathscr{L}_{I_{b}}\left(\mu_{d} r^{\alpha}\left(e^{t}-1\right)\right)=\exp \left(-\pi \lambda r^{2}\left(\beta_{d, b u}\left(e^{t}-1\right)\right)^{2 / \alpha}\right. \\
& \left.\cdot \int_{\left(\beta_{d, b u}\left(e^{t}-1\right)\right)^{-2 / \alpha}}^{\infty} \frac{1}{1+x^{\alpha / 2}} d x\right) .
\end{aligned}
$$

Lemma 2. Laplace transform of $I_{u}$

$$
\begin{aligned}
& \mathscr{L}_{I_{u}}\left(\mu_{d} r^{\alpha}\left(e^{t}-1\right)\right) \\
& =\exp \left(-\pi \lambda r^{2}\left(\beta_{d, u u}\left(e^{t}-1\right)\right)^{2 / \alpha} \int_{0}^{\infty} \frac{1}{1+x^{\alpha / 2}} d x\right) .
\end{aligned}
$$

Proof.

$$
\begin{aligned}
\mathscr{L}_{I_{u}}(s) & =\mathbf{E}_{I_{u}}\left[e^{-s I_{u}}\right] \\
& =\mathbf{E}_{\Omega}\left[\Pi_{i \in \Omega /\left\{u_{o}\right\}} \mathbf{E}_{\varphi_{i}}\left[\exp \left(-s \varphi_{i} R_{i}^{-\alpha}\right)\right]\right] \\
& =\mathbf{E}_{\Omega}\left[\Pi_{i \in \Omega /\left\{u_{o}\right\}} \frac{\kappa_{u u}}{\kappa_{u u}+s R_{i}^{-\alpha}}\right] \\
& =\exp \left(-2 \pi \lambda \int_{0}^{\infty}\left(1-\frac{\kappa_{u u}}{\kappa_{u u}+s v^{-\alpha}}\right) v d v\right) .
\end{aligned}
$$

Therefore,

$$
\begin{aligned}
& \mathscr{L}_{I_{u}}\left(\mu_{d} r^{\alpha}\left(e^{t}-1\right)\right) \\
& =\exp \left(-2 \pi \lambda \int_{0}^{\infty}\left(1-\frac{1}{1+\mu_{d} / \kappa_{u u}\left(e^{t}-1\right)(r / v)^{\alpha}}\right) v d v\right) \\
& \text { where we put } \frac{\mu_{d}}{\kappa_{u u}}=\beta_{d, u u}, \\
& \mathscr{L}_{I_{u}}\left(\mu_{d} r^{\alpha}\left(e^{t}-1\right)\right) \\
& =\exp \left(-2 \pi \lambda \int_{0}^{\infty}\left(1-\frac{1}{1+\beta_{d, u u}\left(e^{t}-1\right)(r / v)^{\alpha}}\right) v d v\right) \\
& =\exp \left(-2 \pi \lambda \int_{0}^{\infty} \frac{1+\beta_{d, u u}\left(e^{t}-1\right)(r / v)^{\alpha}-1}{1+\beta_{d, u u}\left(e^{t}-1\right)(r / v)^{\alpha}} v d v\right) \\
& =\exp \left(-2 \pi \lambda \int_{0}^{\infty} \frac{\beta_{d, u u}\left(e^{t}-1\right)(r / v)^{\alpha}}{1+\beta_{d, u u}\left(e^{t}-1\right)(r / v)^{\alpha}} v d v\right) \\
& =\exp \left(-2 \pi \lambda \int_{0}^{\infty} \frac{\beta_{d, u u}\left(e^{t}-1\right)}{\beta_{d, u u}\left(e^{t}-1\right)+(v / r)^{\alpha}} v d v\right)
\end{aligned}
$$

where we put $\frac{v^{2}}{r^{2}}=u$,

$$
\begin{aligned}
& \mathscr{L}_{I_{u}}\left(\mu_{d} r^{\alpha}\left(e^{t}-1\right)\right) \\
& =\exp \left(-\pi \lambda r^{2} \int_{0}^{\infty} \frac{\beta_{d, u u}\left(e^{t}-1\right)}{\beta_{d, u u}\left(e^{t}-1\right)+u^{\alpha / 2}} d u\right) \\
& =\exp \left(-\pi \lambda r^{2} \int_{0}^{\infty} \frac{1}{1+\left(\left(\beta_{d, u u}\left(e^{t}-1\right)\right)^{-2 / \alpha} u\right)^{\alpha / 2}} d u\right) \\
& \text { where we put }\left(\beta_{d, u u}\left(e^{t}-1\right)\right)^{-2 / \alpha} u=x, \\
& \mathscr{L}_{I_{u}}\left(\mu_{d} r^{\alpha}\left(e^{t}-1\right)\right) \\
& =\exp \left(-\pi \lambda r^{2}\left(\beta_{d, u u}\left(e^{t}-1\right)\right)^{2 / \alpha} \int_{0}^{\infty} \frac{1}{1+x^{\alpha / 2}} d x\right) .
\end{aligned}
$$

4.3. Uplink Rate in Full-Duplex. When a typical UL user $u_{1}$ has a distance $r$ and fading strength $h_{u} \sim \exp \left(\mu_{u}\right)$ to its serving BS, user $u_{1}$ 's signal power at the serving BS is $h_{u} r^{-\alpha}$. Moreover, user $u_{1}$ experiences cumulative interferences $I_{b}$, $I_{u}$, and $I_{s}$. Here, $I_{b}$ is the sum of the received powers from all other BSs, each with distance $r_{i}$ and fading strength $g_{i} \sim$ $\exp \left(\kappa_{b b}\right)$ to the serving BS of user $u_{1}$, such that

$$
I_{b}=\sum_{i \in \Phi_{b}} g_{i} r_{i}^{-\alpha},
$$

where $\Phi_{b}$ is the set of all interfering BSs. Also, $I_{u}$ is the sum of the received powers from all other UL users, each with distance $r_{i}$ and fading strength $\varphi_{i} \sim \exp \left(\kappa_{u b}\right)$ to the serving BS of user $u_{1}$, such that

$$
I_{u}=\sum_{i \in \Omega_{u}} \varphi_{i} r_{i}^{-\alpha},
$$

where $\Omega_{u}$ is the set of all interfering users. Moreover, $I_{s}$ denotes the self-interference by the serving BS of user $u_{1}$. Therefore, the SINR of typical UL user $u_{1}$ can be expressed as

$$
\gamma_{\mathrm{UL}}=\frac{h_{d} r^{-\alpha}}{I_{b}+I_{u}+I_{s}+\sigma^{2}} .
$$

Here, we assume that the self-interference $I_{s}$ amounts to $\varsigma \sigma^{2}$ (i.e., $\varsigma$ times noise power $\sigma^{2}$ ). Then, the average rate of a typical UL user $u_{1}$ is

$$
\begin{aligned}
& R_{\mathrm{UL}}(\lambda, \alpha)=\mathbf{E}_{I_{b}, I_{u}, I_{s}, r}\left[\ln \left(1+\gamma_{\mathrm{UL}}\left(I_{b}, I_{u}, I_{s}, r\right)\right)\right] \\
& =\int_{r>0} e^{-\pi \lambda r^{2}} \\
& \cdot \int_{t>0} e^{-\mu_{u} r^{\alpha}\left(\sigma^{2}+\zeta \sigma^{2}\right)\left(e^{t}-1\right)} \mathscr{L}_{I_{b}}\left(\mu_{u} r^{\alpha}\left(e^{t}-1\right)\right) \\
& \cdot \mathscr{L}_{I_{u}}\left(\mu_{u} r^{\alpha}\left(e^{t}-1\right)\right) d t 2 \pi \lambda r d r .
\end{aligned}
$$


Similar to the downlink analysis, we can obtain the Laplace transform of $I_{b}$ and $I_{u}$ in this uplink case, as follows:

$$
\begin{aligned}
& \mathscr{L}_{I_{b}}\left(\mu_{u} r^{\alpha}\left(e^{t}-1\right)\right) \\
& =\exp \left(-2 \pi \lambda \int_{r}^{\infty}\left(1-\frac{1}{1+\mu_{u} / \kappa_{b b}\left(e^{t}-1\right)(r / v)^{\alpha}}\right)\right. \\
& \cdot v d v) \quad \text { where we put } \frac{\mu_{u}}{\kappa_{b b}}=\beta_{u, b b}, \\
& \mathscr{L}_{I_{b}}\left(\mu_{u} r^{\alpha}\left(e^{t}-1\right)\right) \\
& =\exp \left(-2 \pi \lambda \int_{r}^{\infty} \frac{\beta_{u, b b}\left(e^{t}-1\right)}{\beta_{u, b b}\left(e^{t}-1\right)+(v / r)^{\alpha}} v d v\right) \\
& \mathscr{L}_{I_{b}}\left(\mu_{u} r^{\alpha}\left(e^{t}-1\right)\right) \\
& \quad=\exp \left(-\pi \lambda r^{2} \int_{1}^{\infty} \frac{v^{2}}{1+\left(\left(\beta_{u, b b}\left(e^{t}-1\right)\right)^{-2 / \alpha} u\right)^{\alpha / 2}} d u\right)
\end{aligned}
$$
where we put $\left(\beta_{u, b b}\left(e^{t}-1\right)\right)^{-2 / \alpha} u=x$,

$$
\begin{aligned}
& \mathscr{L}_{I_{b}}\left(\mu_{u} r^{\alpha}\left(e^{t}-1\right)\right)=\exp \left(-\pi \lambda r^{2}\left(\beta_{u, b b}\left(e^{t}-1\right)\right)^{2 / \alpha}\right. \\
& \left.\cdot \int_{\left(\beta_{u, b b}\left(e^{t}-1\right)\right)^{-2 / \alpha}}^{\infty} \frac{1}{1+x^{\alpha / 2}} d x\right), \\
& \mathscr{L}_{I_{u}}\left(\mu_{u} r^{\alpha}\left(e^{t}-1\right)\right) \\
& =\exp \left(-2 \pi \lambda \int_{r}^{\infty}\left(1-\frac{1}{1+\mu_{u} / \kappa_{u b}\left(e^{t}-1\right)(r / v)^{\alpha}}\right)\right. \\
& \cdot v d v) \quad \text { where we put } \frac{\mu_{u}}{\kappa_{u b}}=\beta_{u, u b}, \\
& \mathscr{L}_{I_{u}}\left(\mu_{u} r^{\alpha}\left(e^{t}-1\right)\right) \\
& \quad=\exp \left(-2 \pi \lambda \int_{r}^{\infty} \frac{\beta_{u, u b}\left(e^{t}-1\right)}{\beta_{u, u b}\left(e^{t}-1\right)+(v / r)^{\alpha}} v d v\right) \\
& \text { where we put } \frac{v^{2}}{r^{2}}=u
\end{aligned}
$$

$$
\begin{aligned}
& \mathscr{L}_{I_{u}}\left(\mu_{u} r^{\alpha}\left(e^{t}-1\right)\right) \\
& \quad=\exp \left(-\pi \lambda r^{2} \int_{1}^{\infty} \frac{1}{1+\left(\left(\beta_{u, u b}\left(e^{t}-1\right)\right)^{-2 / \alpha} u\right)^{\alpha / 2}} d u\right)
\end{aligned}
$$$$
\text { where we put }\left(\beta_{u, u b}\left(e^{t}-1\right)\right)^{-2 / \alpha} u=x \text {, }
$$

$$
\begin{gathered}
\mathscr{L}_{I_{u}}\left(\mu_{u} r^{\alpha}\left(e^{t}-1\right)\right)=\exp \left(-\pi \lambda r^{2}\left(\beta_{u, u b}\left(e^{t}-1\right)\right)^{2 / \alpha}\right. \\
\left.\cdot \int_{\left(\beta_{u, u b}\left(e^{t}-1\right)\right)^{-2 / \alpha}}^{\infty} \frac{1}{1+x^{\alpha / 2}} d x\right) .
\end{gathered}
$$

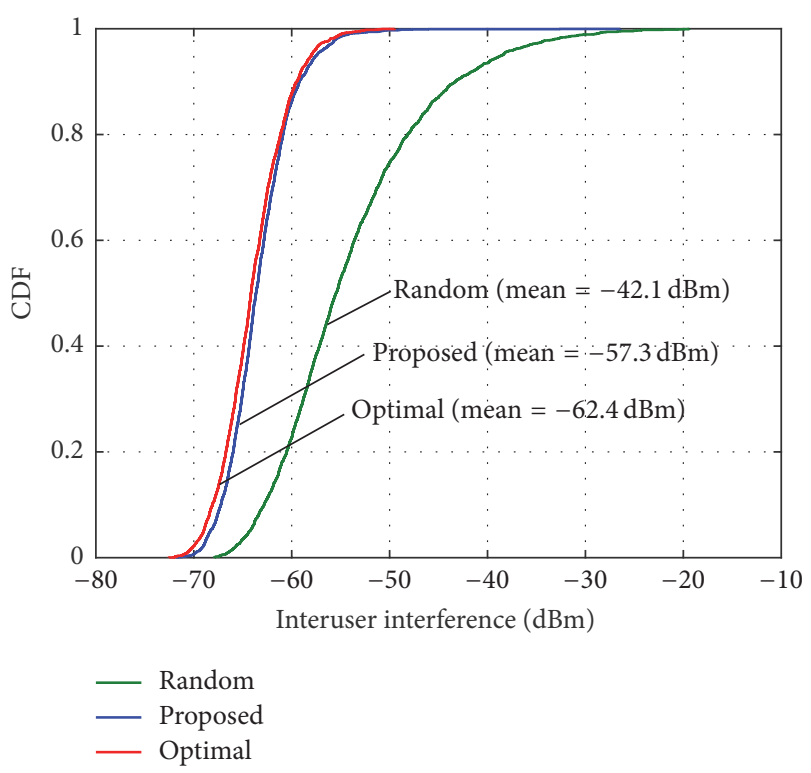

FIGURE 3: Cumulative density function of interuser interference when $\lambda=0.5$.

4.4. Sum Rate in Full-Duplex. The average sum rate, $R_{\mathrm{FD}}(\lambda$, $\alpha$ ), with density $\lambda$ and path loss exponent $\alpha$ is summarized as

$$
R_{\mathrm{FD}}(\lambda, \alpha)=R_{\mathrm{DL}}(\lambda, \alpha)+R_{\mathrm{UL}}(\lambda, \alpha) .
$$

\section{Results and Discussions}

For a performance comparison with the proposed algorithms, we consider (i) the conventional HD mode in which the BS does not use the FD technique, (ii) the FD with the random pairing that determines the user pair randomly, and (iii) the FD with the optimal pairing that determines the optimal user pairs through exhaustive search. We observe the system rate by changing the user density $\lambda$ from 0.1 to 1 . For example, the users with $\lambda=1$ denote the density of one user per $100 \mathrm{~m}^{2}$ on average [24]. For simulation, we consider the same network environment as the one considered at the analysis [10]. The same number of DL and UL users is randomly distributed according to the density $\lambda$ in the multicell network areas. The detailed parameters used for evaluation are listed in Table 1. Here, we obtain the channel parameter from neighbor UL user to UL user, $\kappa_{u u}$, by the simulation after applying each of the considered user pairing algorithms.

Figure 3 plots the cumulative distribution function (CDF) of the interuser interference power when $\lambda=0.5$. This interuser interference level is measured by the simulation when each user pairing algorithm is applied. The distribution of interuser interference is an important measure to verify whether the user pairing algorithm works well. Compared to the random pairing, the proposed pairing remarkably reduces the interuser interference. Moreover, there is no significant difference between the proposed pairing and the optimal pairing. 
TABle 1: Parameter Setup.

\begin{tabular}{lcc}
\hline Parameter & Description & Value \\
\hline$\lambda$ & User density & $0.1 \sim 1($ default $=0.5)$ \\
$\sigma^{2}$ & Noise power & 1 \\
$\alpha$ & Path loss exponent & $4,3,2($ default $=4)$ \\
$\varsigma$ & Self-interference ratio & $0,10,100($ default $=0)$ \\
$\mu_{d}$ & Channel parameter from serving BS to DL user & 0.1 \\
$\mu_{u}$ & Channel parameter from UL user to serving BS & 1 \\
$\kappa_{b u}$ & Channel parameter from neighbor BS to DL user & 0.1 \\
$\kappa_{u b}$ & Channel parameter from neighbor UL user to serving BS & 1 \\
$\kappa_{b b}$ & Channel parameter from neighbor BS to serving BS & $100,1,0.1$ (default $=100)$ \\
$\kappa_{u u}$ & Channel parameter from neighbor UL user to UL user & Variable (obtained by simulation) \\
\hline
\end{tabular}

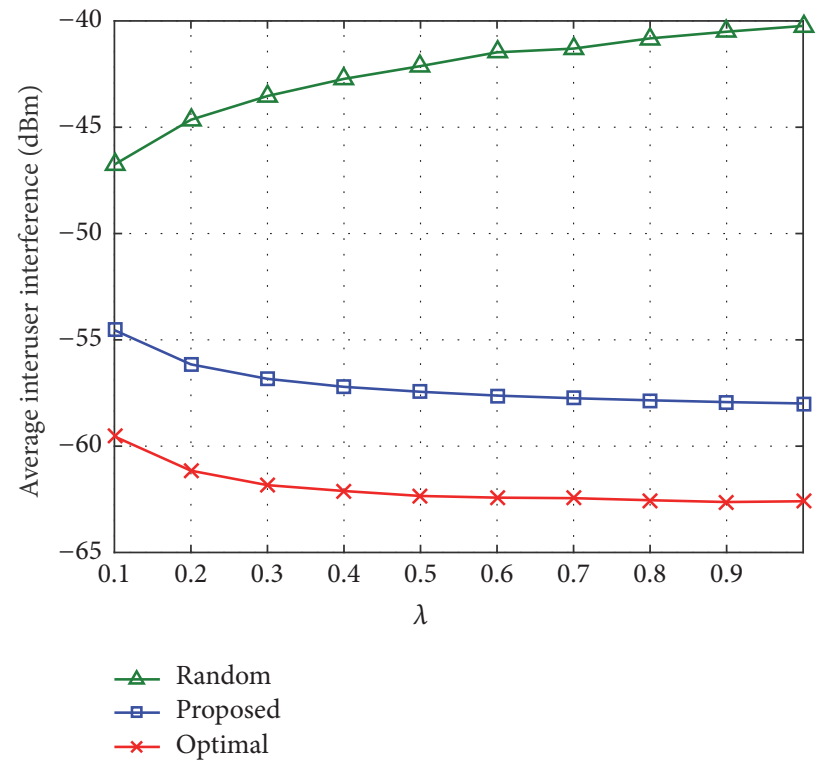

FIgURE 4: Average interuser interference versus user density $\lambda$.

Figure 4 shows the average interuser interference level versus the user density $\lambda$. In the case of the random pairing, the interuser interference increases gradually as the user density increases. This is because the random pairing algorithm often generates uncontrolled stronger interferences between the randomly selected DL and UL users as the number of users increases. On the other hand, in the proposed pairing algorithm, the interuser interference decreases gradually as the user density increases because the user diversity increases as the number of users increases so that the proposed algorithm has more opportunity to select a DL and UL user pair with less interuser interference. The optimal pairing shows the similar tendency as the proposed one and has a slightly lower interuser interference level.

Figure 5 plots the CDF of the uplink rate, downlink rate, and sum rate when $\lambda=0.5$. In the case of the UL rate, the FD mode has the same performance regardless of pairing algorithms because the user pairing algorithm affects only the SINR of DL user. However, the UL rate of FD mode is considerably improved compared with that of HD mode. On the other hand, the DL rate is different according to the pairing algorithm. The DL rate is improved in order of the random, proposed, and optimal pairing because their interuser interference level is reduced in this order, as shown in Figure 4. In the case of the sum rate (i.e., sum of UL and DL rates), the similar performance is shown. We can observe that the rate of the proposed pairing algorithm approaches that of the optimal pairing.

Figure 6 shows the average rate versus the user density $\lambda$. As the user density increases, the sum rate increases because of the effect of user diversity. In each $\lambda$, the UL rates of FD mode are equal regardless of the pairing algorithm. However, the DL rate is different according to the pairing algorithm. The proposed pairing algorithm improves the DL rate greater than the random pairing and approaches the optimal pairing. This verifies the proposed pairing algorithm reduces the interuser interference effectively and so contributes to improve the cell throughput. The performance difference between the proposed pairing and the random pairing increases as $\lambda$ increases because the proposed pairing algorithm has more opportunities to employ the user diversity but the random pairing does not. Compared with the HD mode, the average gains of FD using the random pairing and proposed pairing algorithms are approximately 180 and 195\%, respectively, when $\lambda=0.5$.

Figure 7 shows the average sum rate versus the user density $\lambda$ according to the variations of related parameters: (a) self-interference ratio ( $\varsigma$ ), (b) channel parameter from neighbor BS to serving BS $\left(\kappa_{b b}\right)$, and (c) path loss exponent $(\alpha)$. $\varsigma$ greater than zero means that there remains the selfinterference at the BS using FD. Thus, the sum rates of FD mode decrease as $\varsigma$ increases. In addition, the smaller $\kappa_{b b}$ means that there exists more interference from neighboring BSs because of the imperfect channel estimation. This effect also deteriorates the sum rates of FD mode. When we change the path loss exponent $\alpha$, the sum rate decreases as $\alpha$ decreases and the performance gap between the proposed and random pairing algorithms becomes smaller. This is because the interuser interference increases as the path loss 


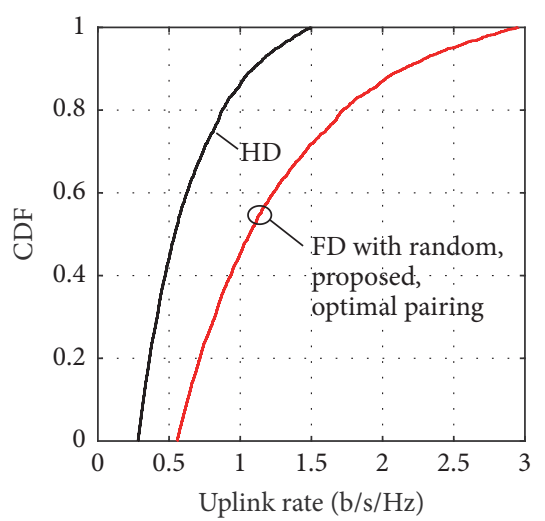

(a)

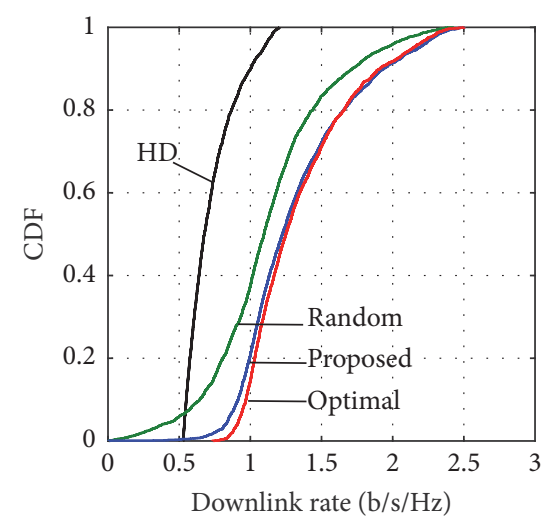

(b)

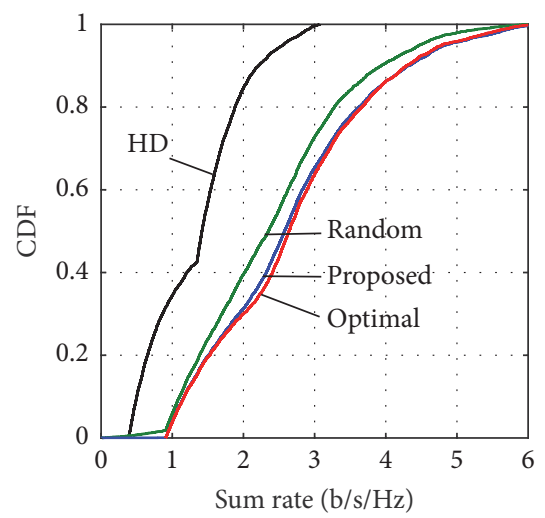

(c)

Figure 5: Cumulative density function of (a) uplink rate, (b) downlink rate, and (c) sum rate when $\lambda=0.5$.

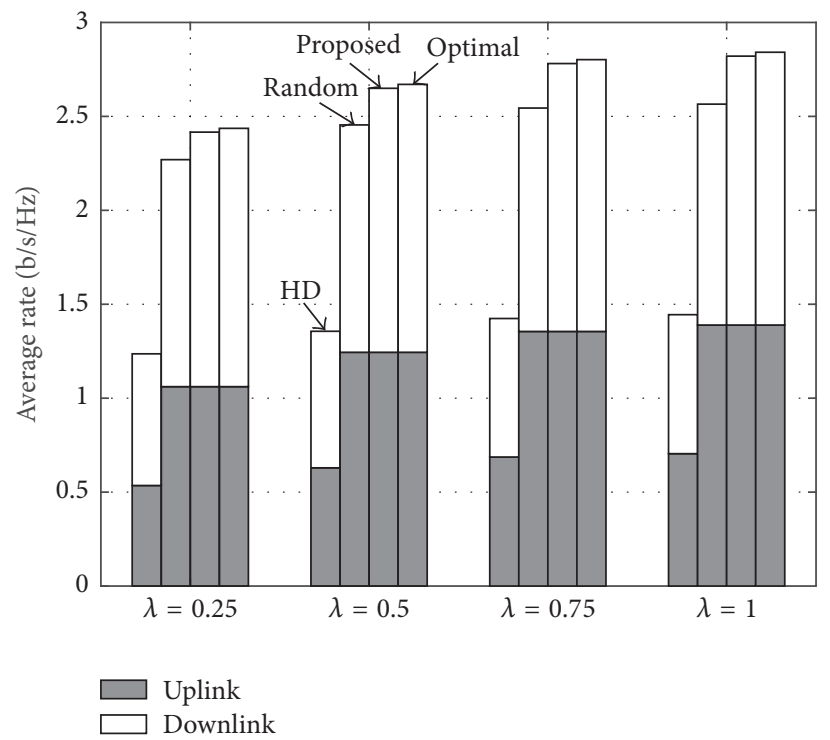

Figure 6: Average rate versus user density $\lambda$.

between users weakens. These results show that the FD system depends heavily on the interference cancellation capability at the BS and it is appropriate to apply in the channel environment with high attenuation in order to maximize its performance.

\section{Conclusion}

In this study, we investigated the issue of user pairing in FD wireless cellular networks. We proposed a heuristic user pairing algorithm with low complexity to maximize cell throughput. The proposed algorithm was designed such that the DL user having a better signal quality has higher priority to choose its UL user for throughput maximization. Then, we analyzed the performance of the FD cellular network theoretically. The results showed that the proposed user pairing algorithm effectively reduced the interuser interference and achieved near-optimal performance. Thus, the FD system that uses it considerably outperforms the FD system using a random user pairing and almost doubles the conventional HD system in terms of cell throughput. For further study, we will extend the proposed user pairing algorithm to more general user scheduling algorithms that dynamically allocate radio resources and the transmission direction in an FD cellular network.

\section{Conflicts of Interest}

The authors declare that there are no conflicts of interest regarding the publication of this paper.

\section{Acknowledgments}

This research was supported by Basic Science Research Program through the National Research Foundation of Korea 


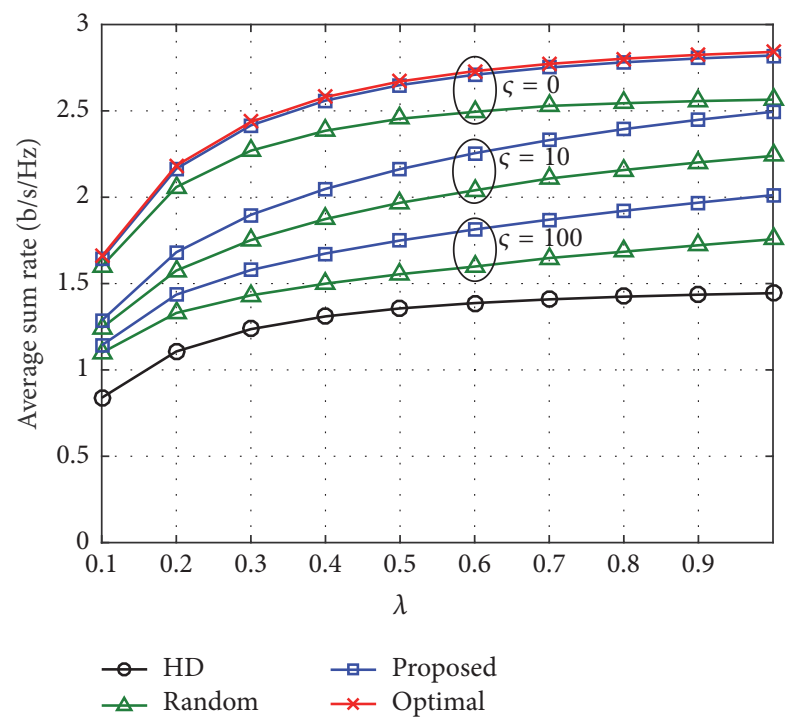

(a)

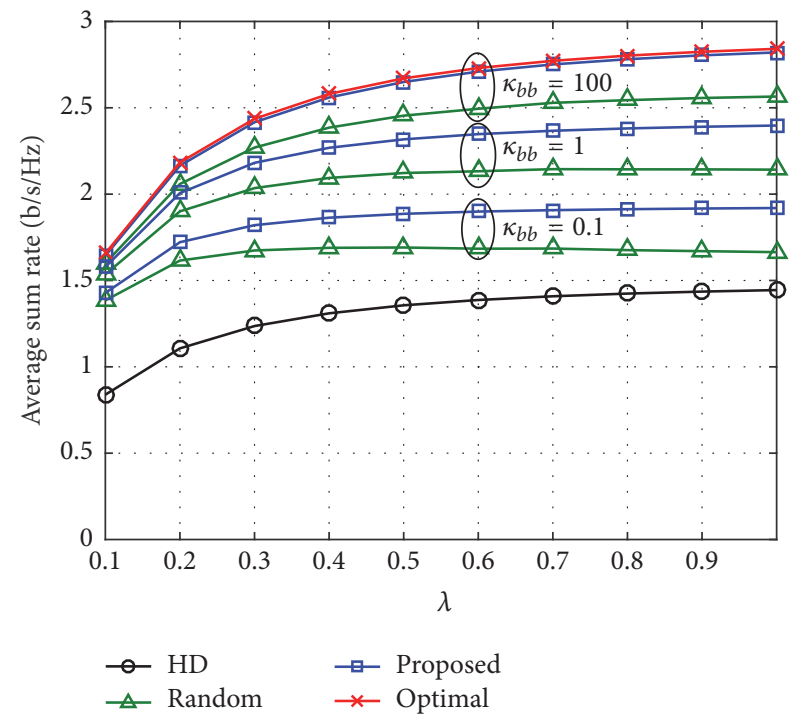

(b)

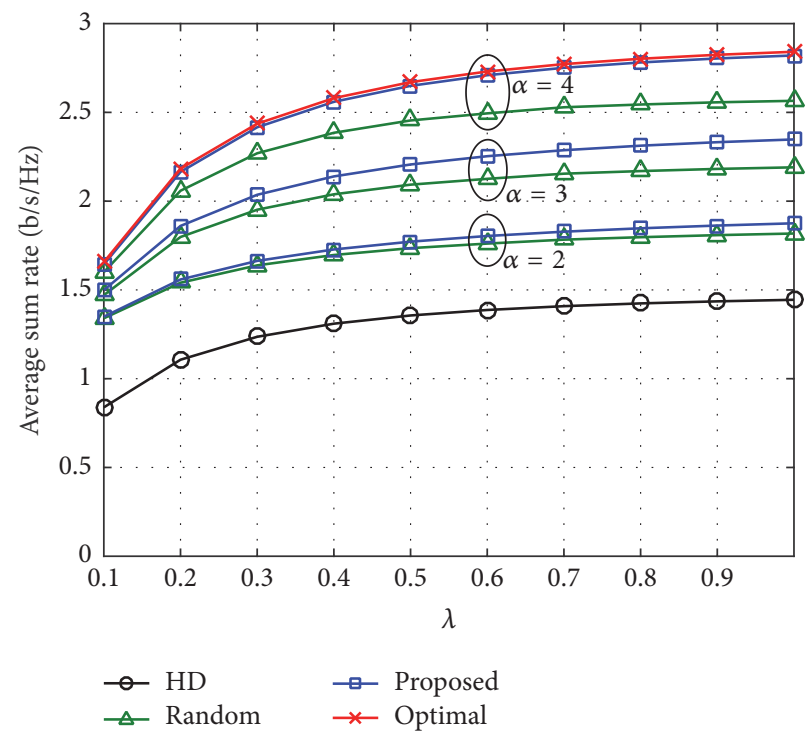

(c)

FIgURE 7: Average sum rate versus user density $\lambda$ according to the variations of (a) $\varsigma$, (b) $\kappa_{b b}$, and (c) $\alpha$.

(NRF) funded by the Ministry of Science, ICT \& Future Planning (NRF-2016R1C1B1016261).

\section{References}

[1] W. C. Y. Lee, “The most spectrum-efficient duplexing system: CDD," IEEE Communications Magazine, vol. 40, no. 3, pp. 163$166,2002$.

[2] W. C. Y. Lee, "CS-OFDMA: a new wireless CDD physical layer scheme," IEEE Communications Magazine, vol. 43, no. 2, pp. 7479, 2005.

[3] X. Jin, M. Ma, B. Jiao, and W. C. Lee, "Studies on spectral efficiency of the CDD system," in Proceedings of the IEEE Vehicular Technology Conference (VTC '09), pp. 1-5, Anchorage, Alaska, USA, September 2009.
[4] J. I. Choi, M. Jain, K. Srinivasan, P. Levis, and S. Katti, "Achieving single channel, full duplex wireless communication," in Proceedings of the 16th Annual International Conference on Mobile Computing and Networking (MobiCom '10), pp. 1-12, IEEE, Chicago, Ill, USA, September 2010.

[5] D. Bharadia, E. McMilin, and S. Katti, "Full duplex radios," in Proceedings of the ACM SIGCOMM Conference on Applications, Technologies, Architectures, and Protocols for Computer Communication (SIGCOMM '13), pp. 375-386, Hong Kong, China, August 2013.

[6] S. Hong, J. Brand, J. Choi II et al., "Applications of selfinterference cancellation in 5G and beyond," IEEE Communications Magazine, vol. 52, no. 2, pp. 114-121, 2014.

[7] D. W. K. Ng and R. Schober, "Dynamic resource allocation in OFDMA systems with full-duplex and hybrid relaying," in 
Proceedings of the IEEE International Conference on Communications (ICC '11), pp. 1-6, Kyoto, Japan, June 2011.

[8] D. W. K. Ng, E. S. Lo, and R. Schober, "Dynamic resource allocation in MIMO-OFDMA systems with full-duplex and hybrid relaying," IEEE Transactions on Communications, vol. 60, no. 5, pp. 1291-1304, 2012.

[9] W. Cheng, X. Zhang, and H. Zhang, "Full/half duplex based resource allocations for statistical quality of service provisioning in wireless relay networks," in Proceedings of the IEEE INFOCOM, pp. 864-872, IEEE, Orlando, Fla, USA, March 2012.

[10] X. Shen, X. Cheng, L. Yang, M. Ma, and B. Jiao, "On the design of the scheduling algorithm for the full duplexing wireless cellular network," in Proceedings of the IEEE Global Communications Conference (GLOBECOM '13), pp. 4970-4975, IEEE, Atlanta, Ga, USA, December 2013.

[11] Z. Liu, Y. Liu, and F. Liu, "Joint resource scheduling for fullduplex cellular system," in Proceedings of the 22nd International Conference on Telecommunications (ICT '15), pp. 85-90, Sydney, Australia, April 2015.

[12] M. Al-Imari, M. Ghoraishi, P. Xiao, and R. Tafazolli, "Game theory based radio resource allocation for full-duplex systems," in Proceedings of the 81st IEEE Vehicular Technology Conference (VTC Spring '15), pp. 1-5, Scotland, UK, May 2015.

[13] Y. Sun, D. W. K. Ng, and R. Schober, "Multi-objective optimization for power efficient full-duplex wireless communication systems," in Proceedings of the 58th IEEE Global Communications Conference (GLOBECOM '15), pp. 1-6, San Diego, Calif, USA, December 2015.

[14] D. Kim, H. Lee, and D. Hong, "A survey of in-band full-duplex transmission: from the perspective of PHY and MAC layers," IEEE Communications Surveys \& Tutorials, vol. 17, no. 4, pp. 2017-2046, 2015.

[15] J. Y. Kim, O. Mashayekhi, H. Qu, M. Kazandjieva, and P. Levis, "Janus: a novel MAC protocol for full duplex radio," Tech. Rep., Stanford University, 2013.

[16] Q. Gao, G. Chen, L. Liao, and Y. Hua, "Full-duplex cooperative transmission scheduling in fast-fading MIMO relaying wireless networks," in Proceedings of the International Conference on Computing, Networking and Communications (ICNC '14), pp. 771-775, Honolulu, Hawaii, USA, February 2014.

[17] G. Yu, D. Wen, and F. Qu, "Joint user scheduling and channel allocation for cellular networks with full duplex base stations," IET Communications, vol. 10, no. 5, pp. 479-486, 2016.

[18] H.-H. Choi, "On the design of user pairing algorithms in full duplexing wireless cellular networks," in Proceedings of the 5th International Conference on Information and Communication Technology Convergence (ICTC '14), pp. 490-495, Busan, Korea, October 2014.

[19] A. Sarkar, P. P. Chakrabarti, and R. Kumar, "Frame-based proportional round-robin," IEEE Transactions on Computers, vol. 55, no. 9, pp. 1121-1129, 2006.

[20] M. Jain, J. Choi, T. Kim et al., "Practical, real-time, full duplex wireless," in Proceedings of the 17th Annual International Conference on Mobile Computing and Networking (ACM MobiCom '11), pp. 301-312, Las Vegas, Nev, USA, September 2011.

[21] H.-H. Choi and J.-R. Lee, "Distributed transmit power control for maximizing end-to-end throughput in wireless multi-hop networks," Wireless Personal Communications, vol. 74, no. 3, pp. 1033-1044, 2014.

[22] J. G. Andrews, F. Baccelli, and R. K. Ganti, "A tractable approach to coverage and rate in cellular networks," IEEE Transactions on Communications, vol. 59, no. 11, pp. 3122-3134, 2011.
[23] T. D. Novlan, H. S. Dhillon, and J. G. Andrews, "Analytical modeling of uplink cellular networks," IEEE Transactions on Wireless Communications, vol. 12, no. 6, pp. 2669-2679, 2013.

[24] S. Goyal, P. Liu, S. Hua, and S. Panwar, "Analyzing a full-duplex cellular system," in Proceedings of the 47th Annual Conference on Information Sciences and Systems (CISS '13), pp. 1-6, IEEE, Baltimore, Md, USA, March 2013. 

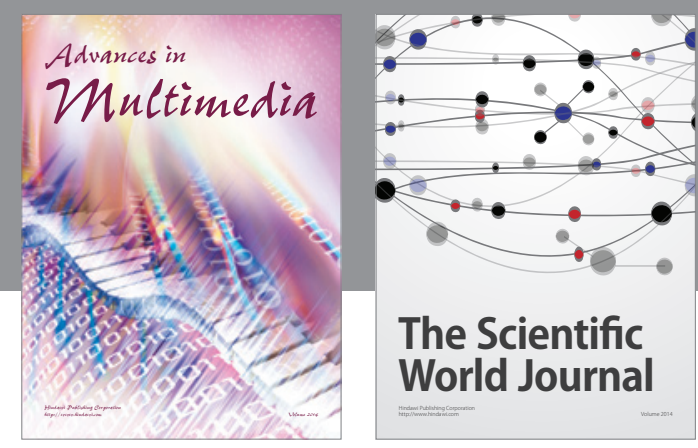

The Scientific World Journal
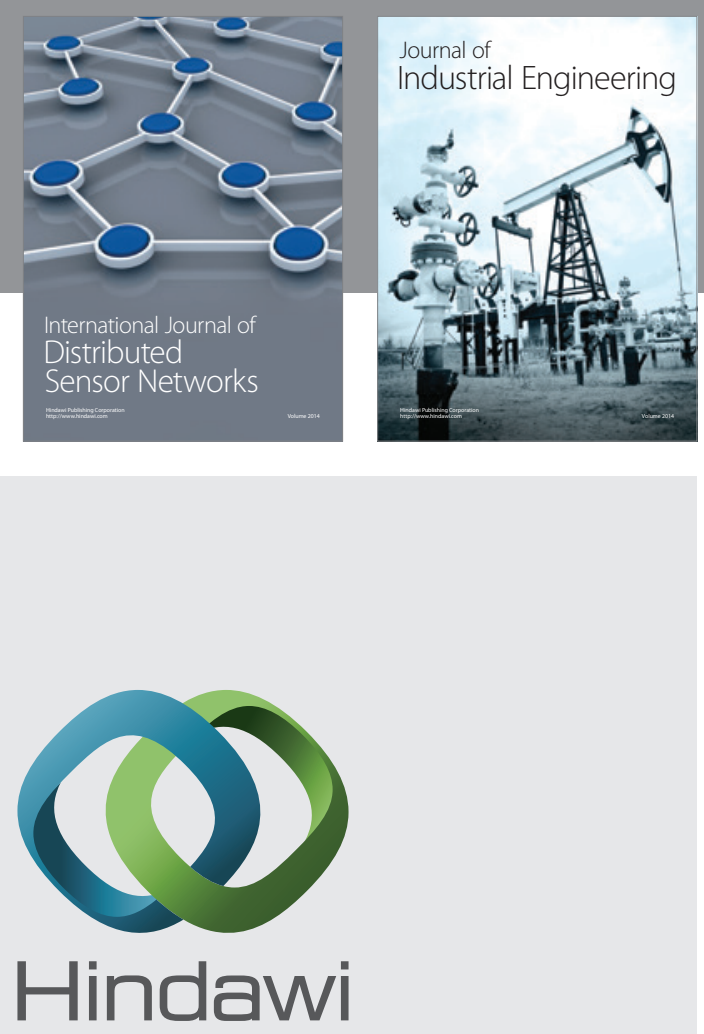

Submit your manuscripts at

https://www.hindawi.com

\section{Computer Networks} and Communications
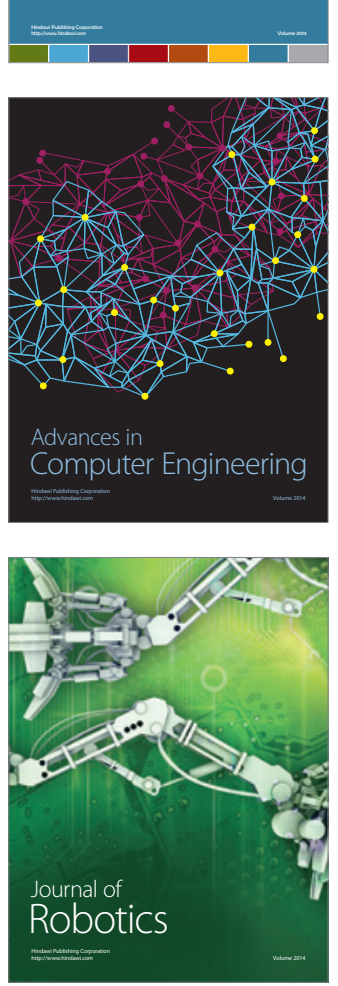
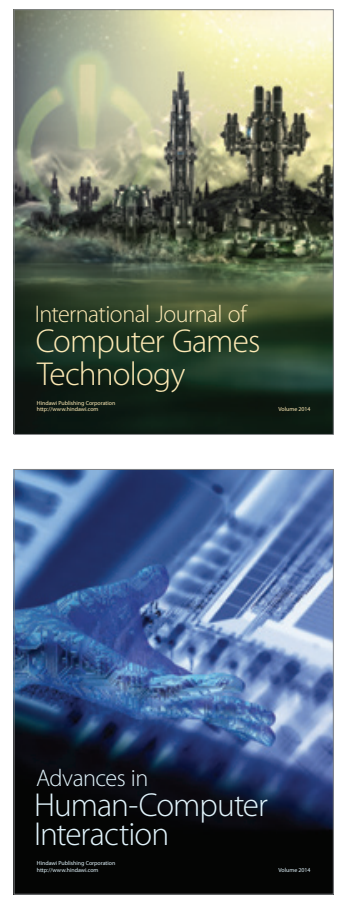
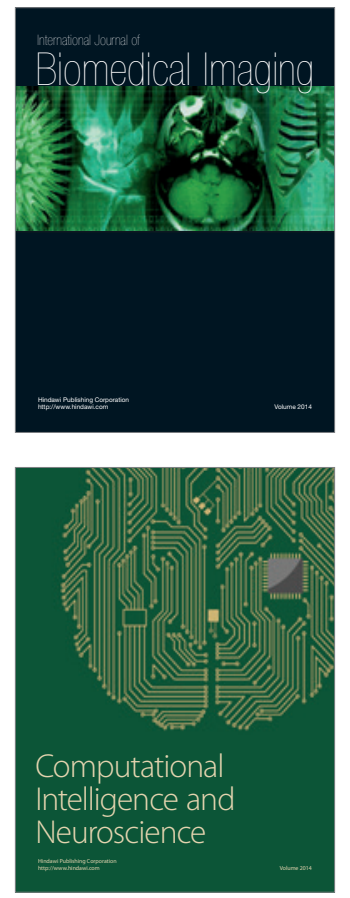
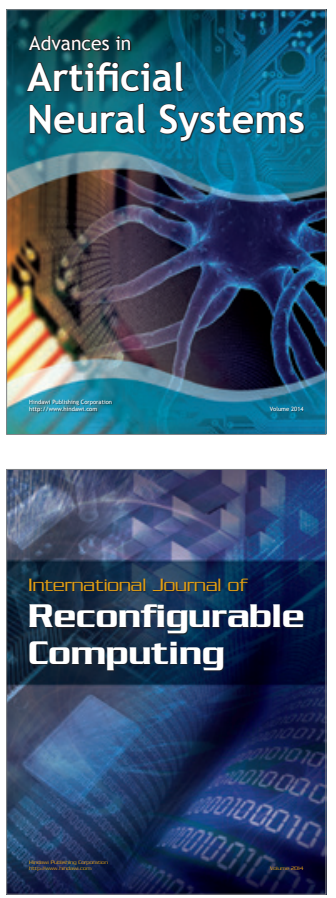
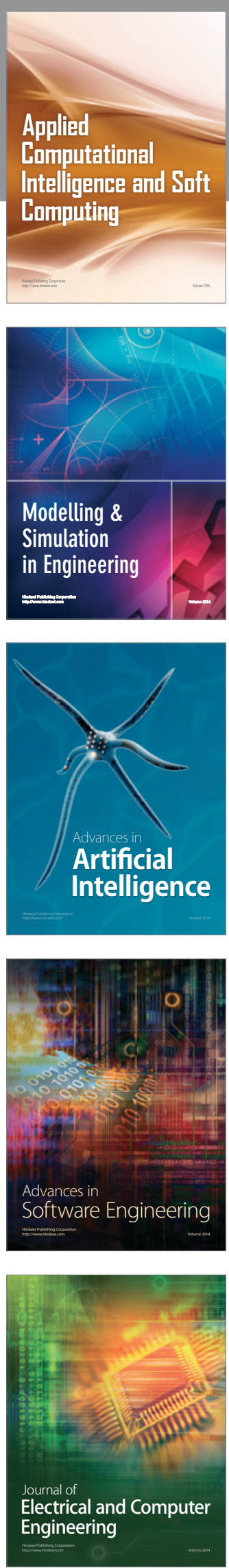